\title{
Isolated fungus ball mimicking mucocele or frontal sinus tumour: a diagnostic pitfall
}

\author{
Popko, M ; Broglie, M A ; Holzmann, D
}

\begin{abstract}
Frontal sinus fungus ball should be considered in the differential diagnosis of chronic, nonspecific forehead symptoms. To evaluate the underlying disease, computed tomography scans should first be performed, followed by magnetic resonance imaging if malignancy is suspected. It is essential to be aware of the possibility of an atypical fungus ball appearance on computed tomography and magnetic resonance imaging scans. If bony destruction and calcification coexist on radiological images, then endoscopic biopsy is an indispensable part of the diagnostic procedure, and should be performed to collect material for both histological and mycological analysis, and to aid surgical planning. In cases of sinus fungus ball, an endoscopic approach for biopsy may be curative.
\end{abstract}

DOI: https://doi.org/10.1017/S0022215109992477

Posted at the Zurich Open Repository and Archive, University of Zurich

ZORA URL: https://doi.org/10.5167/uzh-40423

Journal Article

Published Version

Originally published at:

Popko, M; Broglie, M A; Holzmann, D (2010). Isolated fungus ball mimicking mucocele or frontal sinus tumour: a diagnostic pitfall. Journal of Laryngology and Otology, 124(10):1111-1115.

DOI: https://doi.org/10.1017/S0022215109992477 


\title{
Isolated fungus ball mimicking mucocele or frontal sinus tumour: a diagnostic pitfall
}

\author{
M Popko, M A Broglie*, D HolzmanN*
}

\begin{abstract}
Objective: To demonstrate the clinical, radiological and diagnostic pitfalls of managing an isolated frontal sinus fungus ball, and to compare with the literature.

Material and methods: Retrospective analysis of two cases and literature review.

Results: Isolated frontal sinus fungus ball is a rare cause of frontal sinus disease. We present two cases of isolated frontal sinus fungus ball which pre-operatively were suspected to be either a tumour or a mucocele. In both cases, cheesy, clay-like material was found intra-operatively within the frontal sinus, suggesting a fungus ball. Effective treatment included surgical debridement via an exclusively endoscopic or an external approach, variously. Final histopathological and mycological analysis revealed Aspergillus fumigatus. A literature review revealed 20 reported cases of isolated frontal sinus fungus ball, confirming the low prevalence of the disease.

Conclusions: Frontal sinus fungus ball should be considered in the differential diagnosis of chronic, nonspecific forehead symptoms. To evaluate the underlying disease, computed tomography scans should first be performed, followed by magnetic resonance imaging if malignancy is suspected. It is essential to be aware of the possibility of an atypical fungus ball appearance on computed tomography and magnetic resonance imaging scans. If bony destruction and calcification coexist on radiological images, then endoscopic biopsy is an indispensable part of the diagnostic procedure, and should be performed to collect material for both histological and mycological analysis, and to aid surgical planning. In cases of sinus fungus ball, an endoscopic approach for biopsy may be curative.
\end{abstract}

Key words: Frontal Sinus; Aspergillus Fumigatus; Diagnosis; Imaging

\section{Introduction}

Fungus ball and allergic fungal sinusitis are forms of chronic, non-invasive fungal infection. ${ }^{1}$ Fungus ball of the paranasal sinuses is mostly encountered in older, immunocompetent individuals (average age 64 years), with a female predominance. ${ }^{2}$ The most commonly involved sites are the maxillary sinus ( 94 per cent), followed by the sphenoid sinus (4-8 per cent) and ethmoid sinus (3 per cent). According to Dufour et al., frontal sinus involvement represents only 1.1 per cent of sinus fungus ball cases. ${ }^{2}$

Most patients are either asymptomatic or have nonspecific complaints, i.e. long-lasting frontal headache, fullness in the forehead and upper eyelid region, moderate nasal obstruction, or postnasal drip. The slow, asymptomatic development of frontal sinus fungus ball often leads to late diagnosis, usually with orbital or intracranial complications as a first clinical sign.,

Intra-operative findings of dark brown, cheesy material are highly suggestive of a fungus ball. However, the diagnosis is most accurately made by microscopic detection of fungal material, such as dichotomous, branching hyphae, in smears. The most common pathogens are Aspergillus fumigatus and Scedosporium apiospermum. However, because fungi are fastidious organisms, cultures are negative in up to 50 per cent of cases. ${ }^{5}$
We present two cases of isolated frontal sinus fungus ball which pre-operatively were suspected to be either a mucocele or a tumour. Both cases were successfully treated, exclusively by endoscopic surgery in the first case, and via an external approach in the second (in which a neoplasm was suggested). Additionally, we review the literature on isolated frontal sinus fungus ball.

\section{Materials and methods \\ Patients}

We analysed retrospectively data from two patients referred to the Department of Otolaryngology Head and Neck Surgery, University Hospital of Zurich. These patients were suspected of having a frontal sinus tumour or mucocele.

\section{Literature review}

We searched the PubMed and Medline databases from January 1973 to October 2008 for all relevant English, German and Polish language studies. The following Medical Subject Headings and search terms were used: (mycetoma and paranasal sinuses), (frontal sinus fungus

From the Department of Otolaryngology, Poznan University of Medical Science, Poland, and the *Department of Otolaryngology Head and Neck Surgery, University Hospital of Zurich, Switzerland.

Accepted for publication: 21 September 2009. First published online 23 December 2009. 
ball), (aspergillosis frontal sinus), (aspergilloma frontal sinus) and (frontal sinus mass).

\section{Results}

Case one

A 74-year-old, immunocompetent man presented with a sixmonth history of moderate nasal obstruction, postnasal drip and a pressure sensation over the left side of the forehead, with moderate pain especially in the morning following a common cold. He had been treated for chronic rhinosinusitis with antibiotics and local decongestants. As his condition did not improve, he was referred to our hospital.

Nasal endoscopy showed no pathology.

Computed tomography (CT) scanning revealed a leftsided, heterogeneous, fronto-ethmoidal recess opacity and central hypo-attenuation, with bilateral frontal sinus and left anterior ethmoidal cell extension, as well as erosion and sclerosis of the surrounding bone, but no evidence of intracranial expansion (Figure 1). Magnetic resonance imaging (MRI) showed expansive opacity in both frontal sinuses, the left fronto-ethmoidal recess and the anterior ethmoidal cells. There was peripheral linear enhancement and central hypointense signal on T1- and T2-weighted images, with no evidence of intracranial expansion (Figure 2). Both CT and gadolinium-enhanced MRI scans showed border enhancement of the mass suggesting the neoplasm or mucocele.

The patient was selected for an explorative endoscopic biopsy to evaluate suspected malignancy. Intra-operatively, cheesy, clay-like material was found within the frontal sinus, highly suggestive of a sinus fungus ball. Frozen section analysis confirmed fungal hyphae and inflammatory sinus disease. An endoscopic left fronto-ethmoidectomy with endonasal Draf type II frontal sinusotomy was performed, achieving complete removal of the mass.

The removed material was sent for routine histopathological and comprehensive microbiological analysis.

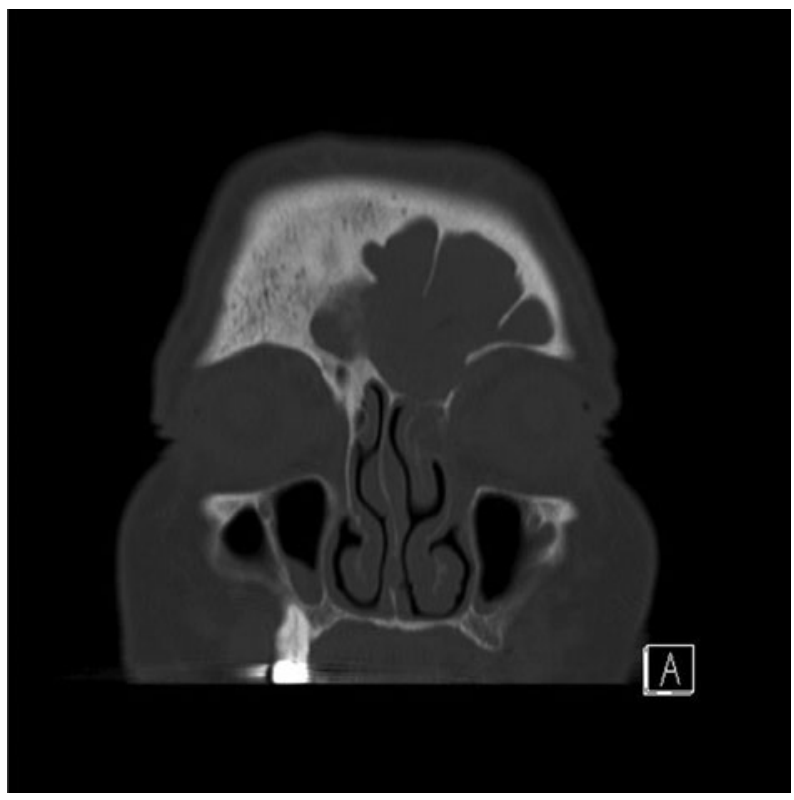

FIG. 1

Case one: coronal computed tomography scan showing a left-sided, heterogeneous, fronto-ethmoidal recess opacity with central hypoattenuation, with bilateral frontal sinus and left anterior ethmoidal cell extension, accompanied by erosion and sclerosis of the surrounding bone but no evidence of intracranial expansion. $\mathrm{A}=$ anterior, $\mathrm{L}=$ left side

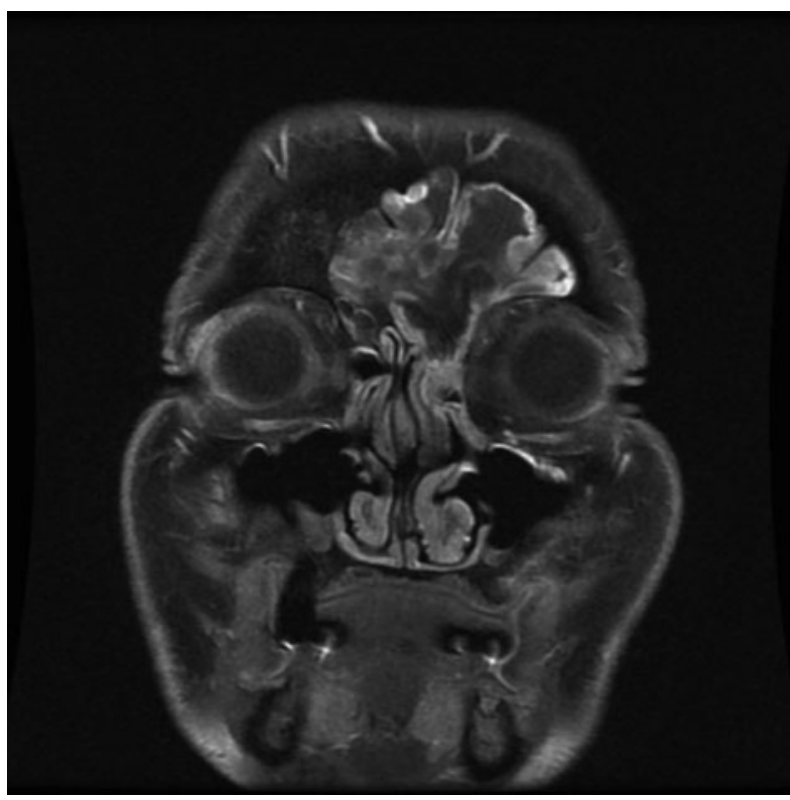

FIG. 2

Case one: coronal, T1-weighted magnetic resonance imaging scan showing expansive opacity in the bilateral frontal sinus, left fronto-ethmoidal recess and anterior ethmoidal cells, with peripheral linear enhancement and central hypointense signal, and no evidence of intracranial expansion.

Macroscopic examination showed brown-beige, bulbous, friable tissue fragments. Microscopic examination demonstrated chronic inflammation with diffuse fibrosis, septae and $45^{\circ}$, dichotomous, branching hyphae, establishing a diagnosis of aspergillus. Cultures grew $A$ fumigatus.

At eight months' follow up, the patient was symptom-free.

\section{Case two}

A 63-year-old man presented with a two-year history of frontal facial pain worse on the right, aggravated within the last two months, and postnasal drip.

During ENT examination, the patient reported tenderness of the right frontal region on palpation.

Nasal endoscopy revealed no pathology.

Computed tomography and MRI scanning of the head showed bilateral, heterogeneous frontal sinus opacity, with calcifications in the base of the left frontal sinus and an eroded posterior frontal sinus wall, interfrontal sinus septum and right orbital roof (Figures 3 and 4). Radiologists suspected a sinus fungus ball; however, due to the advanced bony erosion, malignant disease could not be excluded.

As a neoplasm was suggested, and due to the large defect in the posterior wall of the frontal sinus, surgical exploration with fat obliteration was indicated, through an external approach via coronal incision. Intra-operatively, brownish, cheesy debris were found within the sinus.

Frozen section analysis and definitive histopathological examination were both consistent with an aspergillus fungus ball, and $A$ fumigatus was cultured.

At 15 months' follow up, the patient was disease-free without sequelae.

\section{Literature review}

Table I shows reports of isolated frontal sinus fungus ball published in the last 25 years. 


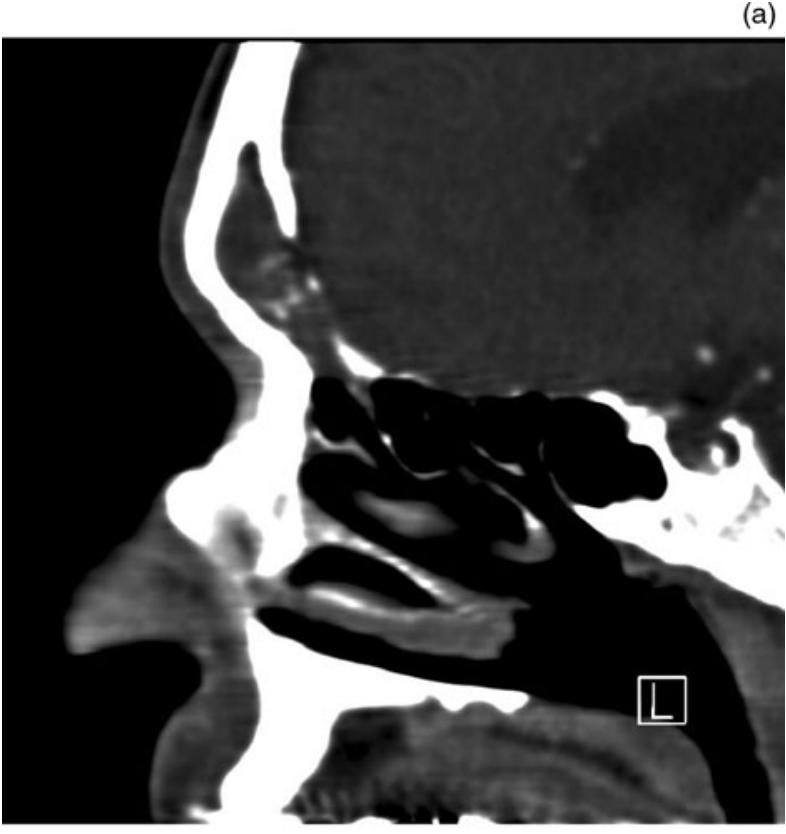

(b)

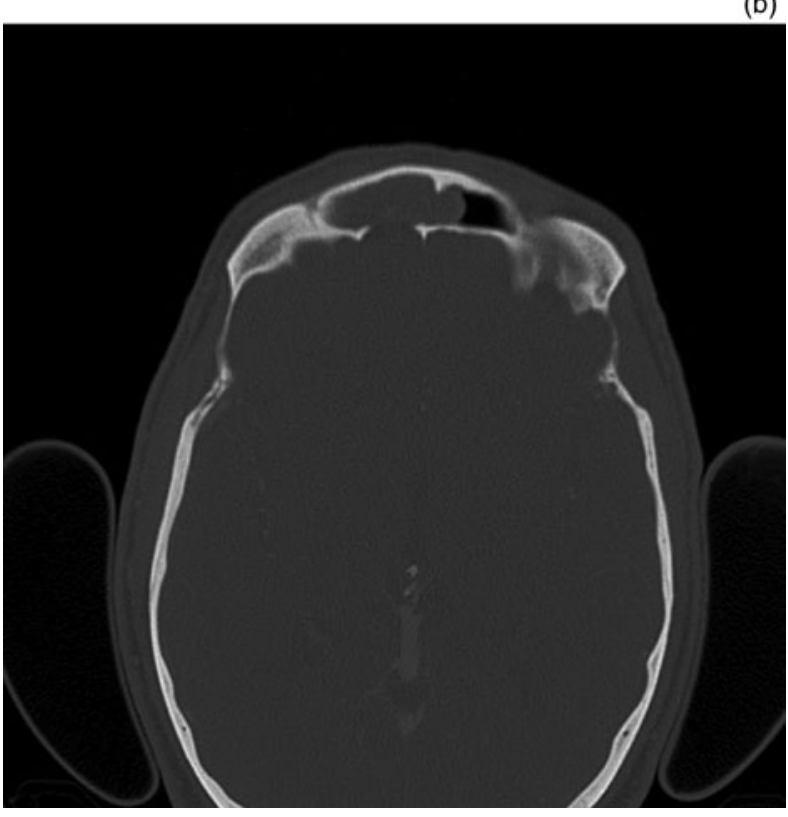

FIG. 3

Case two: (a) sagittal and (b) axial computed tomography scans of the head, showing bilateral, heterogeneous frontal sinus opacification, with calcifications in the base of the left frontal sinus and an eroded posterior frontal sinus wall, interfrontal sinus septum and right orbital roof. $\mathrm{A}=$ anterior, $\mathrm{L}=$ left side

\section{Discussion}

At our institution, isolated frontal sinus fungus balls have previously been calculated to account for 2 per cent of paranasal sinus fungus ball cases. ${ }^{18}$ This finding is similar to that of Klossek et al., who found that frontal sinus involvement represented only approximately 2 per cent of all cases of paranasal sinus fungus ball. ${ }^{11}$ Dufour et al. recently reported an incidence of 1.1 per cent. ${ }^{2}$ Such rarity can be explained by poor access to the frontal sinus for the mainstream of inhaled spores. ${ }^{4}$ Our literature review identified only 20 cases of isolated frontal sinus fungus ball, reflecting the rarity of this disease.

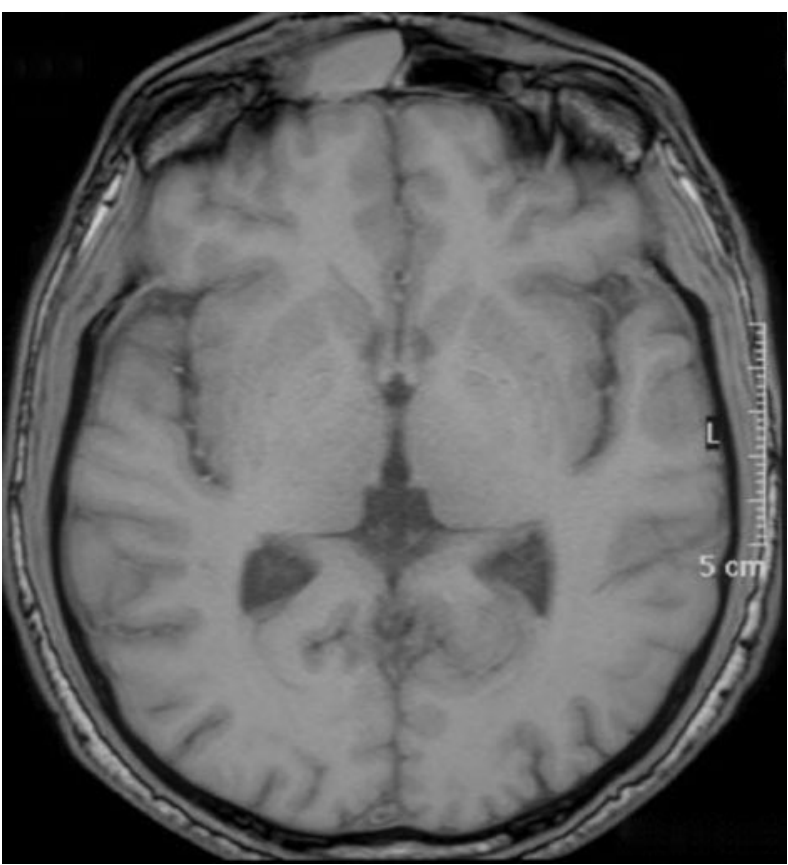

FIG. 4

Case two: axial, T1-weighted magnetic resonance imaging scan showing opacity in the right frontal sinus, with no evidence of intracranial expansion. $\mathrm{A}=$ anterior, $\mathrm{L}=$ left side

Isolated frontal sinus fungus balls generally remain asymptomatic until complications occur. Orbital or intracranial involvement commonly causes the first clinical signs. $^{4}$ Accordingly, our patients presented with nonspecific symptoms. A diagnosis of chronic sinusitis was suggested by our first patient's persistent frontal headache, fullness in the forehead and upper eyelid region, moderate nasal obstruction, and postnasal drip, and by our second patient's frontal headache and postnasal drip. Gerlinger et al. reported that malignant frontal sinus tumours may mimic inflammatory disease. ${ }^{19}$ Frontal sinus tumours most commonly present with vague symptoms such as forehead swelling, pain and visual disturbance. Occasionally, watery rhinorrhoea, purulent nasal discharge, mental confusion and convulsions can occur.

Our first patient's symptoms were considered to indicate a possible frontal sinus mucocele, since frontal headache, fullness in the forehead and upper eyelid region, nasal

TABLE I

PREVIOUS REPORTS OF ISOLATED FRONTAL SINUS FUNGUS BALL

\begin{tabular}{|c|c|}
\hline Study & Cases $(n)$ \\
\hline Babinski et al. ${ }^{6}$ & 1 \\
\hline Bhalla et al. & 1 \\
\hline Chen \& Chen ${ }^{8}$ & 1 \\
\hline Dufour et $a l^{2}$ & 4 \\
\hline Ferreiro et al. ${ }^{9}$ & 2 \\
\hline Gupta et al. ${ }^{10}$ & 1 \\
\hline Klossek et al. ${ }^{11}$ & 2 \\
\hline Kodama et al. ${ }^{12}$ & 1 \\
\hline Kumar et al. ${ }^{13}$ & 1 \\
\hline Panda \& Reddy ${ }^{14}$ & 2 \\
\hline Sekula et al. ${ }^{15}$ & 1 \\
\hline Stevens $^{16}$ & 1 \\
\hline Swoboda \& Ullrich ${ }^{4}$ & 1 \\
\hline Warder et al. ${ }^{17}$ & 1 \\
\hline Total & 20 \\
\hline
\end{tabular}


discharge, and obstruction are the most frequent clinical manifestations of this disease. ${ }^{20}$ However, this patient's absence of previous trauma or surgery made this diagnosis unlikely.

On CT scanning, 90 per cent of frontal sinus fungus balls show partial to complete, heterogeneous, central opacification of the frontal sinus, with a zone of reduced opacity between the central area and the bony sinus wall. Less commonly (in 10 per cent), a homogeneous opacification is observed. Additionally, microcalcifications and/or 'metallic dense spots' (i.e. areas of hyperattenuation) associated with sclerosis of the bony wall imply the presence of a fungus ball. ${ }^{1}$ Magnetic resonance images display an intermediate to hypointense signal on T1-weighted images and an iso- or hypointense signal, or even no signal, on T2-weighted images. ${ }^{3}$

Although the diagnostic criteria defined by Shazo et al., based on clinical, radiological and histological examination, are said to be highly pathognomonic, in our two cases pre-operative radiological evaluation did not lead to the correct diagnosis. ${ }^{1}$ Computed tomography and MRI scans did not show a partial or complete, heterogeneous, central opacity in our first case. This patient's images demonstrated bone erosion, sclerosis and no sign of calcification, in contrast to reports stating that fungus balls appear as soft tissue masses with calcification and without bone erosion. ${ }^{19}$ Our second patient's CT scan showed basal calcification but advanced bone erosion, prompting suspicion of a malignancy.

- In cases of chronic, nonspecific symptoms unresponsive to antibiotic treatment, sinus fungus ball should be considered in the differential diagnosis

- To evaluate underlying disease, computed tomography (CT) should first be performed, followed by magnetic resonance imaging (MRI) if malignancy is suspected

- It is essential to be aware of the possibility of an atypical fungus ball appearance on CT and MRI

- If bony destruction and calcification coexist on radiological images, endoscopic biopsy must be included in the diagnostic procedure to collect material for histological and mycological investigation

According to Rao et al., CT images of both mucoceles (i.e. mucoid-filled sinuses resulting from ostial obstruction caused by inflammatory scarring, trauma or tumour) and frontal sinus tumours demonstrate an expanded sinus with gradual thinning and erosion of the bony margins. ${ }^{21}$ Magnetic resonance imaging of mucoceles characteristically reveals a thin, peripheral, linear enhancement with central, low signal intensity on T1-weighted images. Interestingly, these findings were present in our first case. On the other hand, frontal sinus malignancies show diffuse, intermediate signal intensity on T1- and T2-weighted MRI scans. Although MRI plays a critical role in differentiating tumours from surrounding soft tissue, inflammatory disease and retained secretions within the sinus, our first patient's diagnosis remained unreliable following radiological examination. His condition was misinterpreted as mucocele neoplasm. Magnetic resonance imaging was also performed in our second case but it was assumed that the advanced bone destruction seen on CT indicated clearly a malignant process. If the pre-operative MRI rather than CT had been considered in this patient, endoscopic biopsy may have been undertaken as the initial surgical procedure. The intra-operative discovery of cheesy, clay-like material would then have prompted suspicion of a sinus fungus ball, which subsequently could have been completely removed endoscopically. Histological and mycological confirmation of the diagnosis could then have prevented open surgery.

Our first patient was scheduled to undergo exploratory endoscopy with biopsy. Exclusively endoscopic surgery was successfully performed, with complete removal of the fungus ball. To our knowledge, Kodama et al. were the first to introduce a purely endoscopic treatment for frontal sinus fungus ball. ${ }^{12}$

Surprisingly, intra-operative exploration in both our patients revealed cheesy, clay-like material highly suggestive of a fungus ball. Accurate diagnosis was confirmed by histological investigation and isolation of $A$ fumigatus from cultures. According to the literature, only 23-50 per cent of fungus ball cultures are positive; those that are usually grow $A$ fumigatus, and occasionally $S$ apiospermum (Pseudoallescheria boydii), A flavus, A niger or A terrus. ${ }^{5}$

\section{Conclusion}

Our two cases did not show the expected imaging signs reported in the literature. The typical imaging characteristics of fungus ball are considered important criteria defining this entity. However, our patients' radiological findings did not correspond with their final diagnosis, being misinterpreted as indicating a malignant process or mucocele. The intra-operative appearance, on the other hand, was pathognomonic in both cases, leading to the correct diagnosis.

We therefore suggest that: (1) in every case of chronic, nonspecific symptoms unresponsive to antibiotic treatment, sinus fungus ball should be considered in the differential diagnosis; (2) to evaluate the underlying disease, CT should be performed first, followed by MRI if malignancy is suspected; (3) clinicians should be aware of atypical fungus ball appearances on CT and MRI; (4) if bony destruction and calcification coexist in radiological images, endoscopic biopsy must be included in the diagnostic procedure to collect material for both histological and mycological investigation. Such an approach will either be curative in itself or will assist planning of subsequent treatment.

Our findings indicate that, although diagnostic criteria for paranasal fungus ball have been proposed, correct clinical and radiological diagnosis of isolated frontal sinus fungus ball still remains a challenge.

\section{References}

1 deShazo RD, O'Brien M, Chapin K, Soto-Aguilar M, Swain R, Lyons $\mathrm{M}$ et al. Criteria for the diagnosis of sinus mycetoma. J Allergy Clin Immunol 1997;99:475-85

2 Dufour X, Kauffmann-Lacroix C, Ferrie JC, Goujon JM, Rodier MH, Karkas A et al. Paranasal sinus fungus ball and surgery: a review of 175 cases. Rhinology 2005;43: 34-9

3 Grosjean P, Weber R. Fungus ball of the paranasal sinuses: a review. Eur Arch Otorhinolaryngol 2007:264:461-70

4 Swoboda H, Ullrich R. Aspergilloma in the frontal sinus expanding into the orbit. J Clin Pathol 1992;45:629-30

5 Ferguson BJ. Fungus ball of the paranasal sinuses. Otolaryngol Clin North Am 2000;33:389-98

6 Babinski D, Narozny W, Skorek A, Rzepko R, Stankiewicz C. Noninvasive fungal sinusitis (fungus ball) - diagnostic difficulties [in Polish]. Otolaryngol Pol 2007;61:694-7

7 Bhalla D, Kumar S, Pal ND, Malhotra V, Dhingra PL. Aspergilloma of the frontal lobe. Acta Neurochir 1980;55: $135-9$ 
8 Chen H, Chen TM. Isolated frontal sinus aspergillosis. Otolaryngol Head Neck Surg 2000;122:460-1

9 Ferreiro JA, Carlson BA, Cody DT. Paranasal sinus fungus balls. Head Neck 1997;19:481-6

10 Gupta KR, Udhayakumar S, Rao PB, Madhaven M, Das Gupta LR. Aspergilloma of the frontal bone. J Laryngol Otol 1973;87:1007-11

11 Klossek JM, Serrano E, Péloquin L, Percodani J, Fontanel JP, Pessey JJ. Functional endoscopic sinus surgery and 109 mycetomas of the paranasal sinuses. Laryngoscope 1997 107:112-17

12 Kodama S, Moriyama M, Okamoto T, Hirano T, Suzuki M. Isolated frontal sinus aspergillosis treated by endoscopic modified Lothrop procedure. Auris Nasus Larynx 2009; 36:88-91

13 Kumar BS, Patro M, Mishra D, Bal A, Behera B, Sahoo S. Fine needle aspiration in aspergilloma of frontal sinus: a case report. Acta Cytol 2008;52:500-4

14 Panda NK, Reddy CEE. Primary frontal sinus aspergillosis: an uncommon occurrence. Mycoses 2005;48:235-7

15 Sekula J, Popielski L, Betkowski A. A case of aspergillosis involving the frontal sinus and orbit [in Polish]. Otolaryngol Pol 1981;35:365-9

16 Stevens MH. Aspergillosis of the frontal sinus. Arch Otolaryngol 1978;104:153-6

17 Warder FR, Chikes PG, Hudson WR. Aspergillosis of the paranasal sinuses. Arch Otolaryngol 1975;101:683-5

18 Broglie MA, Tinguely M, Holzmann D. How to diagnose sinus fungus balls in the paranasal sinus? An analysis of an institution's cases from 1999 - 2006. Rhinology 2009, in press

19 Gerlinger I, Gobel G, Toth E, Szanyi I, Weninger C. Primary carcinoma of the frontal sinus: a case report and review of literature. Eur Arch Otolaryngol 2008;265: 593-7

20 Mourouzis C, Evans BT, Shenouda E. Late presentation of mucocele of the frontal sinus: 50 years postinjury. $J$ Oral Maxillofac Surg 2008;66:1510-13

21 Rao MV, Sharma D, Madan A. Imaging of frontal sinus disease. Otolaryngol Clin North Am 2001;34:23-39

Address for correspondence:

Dr Mariola Popko,

Department of Otolaryngology,

Poznan University of Medical Science,

Przybyszewskiego 49,

60-355 Poznan, Poland

Fax: +48 618691690

E-mail: popkom@interia.pl

Dr M Popko takes responsibility for the integrity of the content of the paper.

Competing interests: None declared 